\title{
An alternating active-dormitive strategy enables disadvantaged prey to outcompete the perennially active prey through Parrondo's paradox
}

\author{
Tao Wen ${ }^{1}$, Eugene V. Koonin ${ }^{2}$ and Kang Hao Cheong ${ }^{1 *}$ (D)
}

\begin{abstract}
Background: Dormancy is widespread in nature, but while it can be an effective adaptive strategy in fluctuating environments, the dormant forms are costly due to the inability to breed and the relatively high energy consumption. We explore mathematical models of predator-prey systems, in order to assess whether dormancy can be an effective adaptive strategy to outcompete perennially active (PA) prey, even when both forms of the dormitive prey (active and dormant) are individually disadvantaged.

Results: We develop a dynamic population model by introducing an additional dormitive prey population to the existing predator-prey model which can be active (active form) and enter dormancy (dormant form). In this model, both forms of the dormitive prey are individually at a disadvantage compared to the PA prey and thus would go extinct due to their low growth rate, energy waste on the production of dormant prey, and the inability of the latter to grow autonomously. However, the dormitive prey can paradoxically outcompete the PA prey with superior traits and even cause its extinction by alternating between the two losing strategies. We observed higher fitness of the dormitive prey in rich environments because a large predator population in a rich environment cannot be supported by the prey without adopting an evasive strategy, that is, dormancy. In such environments, populations experience large-scale fluctuations, which can be survived by dormitive but not by PA prey.
\end{abstract}

Conclusion: We show that dormancy can be an effective adaptive strategy to outcompete superior prey, recapitulating the game-theoretic Parrondo's paradox, where two losing strategies combine to achieve a winning outcome. We suggest that the species with the ability to switch between the active and dormant forms can dominate communities via competitive exclusion.

Keywords: Parrondo's paradox, Population dynamics, Predator-prey, Prey dormancy

\footnotetext{
* Correspondence: kanghao_cheong@sutd.edu.sg

${ }^{1}$ Science, Mathematics and Technology Cluster, Singapore University of

Technology and Design (SUTD), 8 Somapah Road, S487372, Singapore,

Singapore

Full list of author information is available at the end of the article
}

C C The Author(s). 2021 Open Access This article is licensed under a Creative Commons Attribution 4.0 International License, which permits use, sharing, adaptation, distribution and reproduction in any medium or format, as long as you give appropriate credit to the original author(s) and the source, provide a link to the Creative Commons licence, and indicate if changes were made. The images or other third party material in this article are included in the article's Creative Commons licence, unless indicated otherwise in a credit line to the material. If material is not included in the article's Creative Commons licence and your intended use is not permitted by statutory regulation or exceeds the permitted use, you will need to obtain permission directly from the copyright holder. To view a copy of this licence, visit http://creativecommons.org/licenses/by/4.0/ The Creative Commons Public Domain Dedication waiver (http://creativecommons.org/publicdomain/zero/1.0/) applies to the data made available in this article, unless otherwise stated in a credit line to the data. 


\section{Background}

Dormancy, such as hibernation in mammals $[1,2]$, diapause of insects and zooplankton [3], as well as the soil seed banks $[4,5]$, is a widespread and efficient adaptive strategy for populations under environmental fluctuation. Various types of dormancy are also a common form of stress response in bacteria including sporulation of Gram-positive bacteria [6-8]. The evolutionary stability of dormancy has been investigated under varying environments $[3,9,10]$, as well as under the environment with minimal abiotic fluctuations [3]. However, the dormant form of an organism is much more costly compared with the active form in the course of evolution, due to its inability to breed and the large energy consumption during resting egg production as is the case for many invertebrates, such as zooplankton [11, 12].

Hence, dormancy has become a topic of major interest in evolutionary biology: why does dormancy persist and remain a competitive evolutionary strategy? Previous studies have shown the advantage of predator dormancy when two types of predators compete for a single resource [13, 14]. This phenomenon was first experimentally discovered by [15] and subsequently analyzed by [16]. They found that large-amplitude fluctuations can be avoided by predator dormancy in the predator-prey dynamic population model, explaining the paradox of enrichment [17-19]. However, the effect of prey dormancy has not been studied: how will dormancy affect the prey, and can prey dormancy also suppress largeamplitude fluctuation?

In order to identify the factors that determine whether prey remains active or goes into dormancy, it is necessary to quantify the competition among prey. Under the high density of predators, the conditions are harsh for prey because they have to avoid being foraged by numerous predators. Under these conditions, the ability to enter dormancy will be beneficial to the prey allowing it to reduce its consumption and breeding, as well as the probability of being discovered. In contrast, the prey will remain active to promote population growth under a low density of predators. Furthermore, dormancy is beneficial for prey (actually, for any organism) under large-amplitude environmental fluctuation, especially under harsh environmental conditions, but not in a safe, stable environment. Recently, it has been shown that environmentally destructive populations can survive by switching between "nomadic" and "colonial" forms [2022]. Predator dormancy also allows the predator to survive in the large-amplitude fluctuation [14]. In these models, one form grows rapidly but depletes the environment (which includes prey in the case of predator and host in the case of a parasite), whereas the other one does not affect the environment but decays. Each of these strategies individually results in extinction, but combined, they can ensure the survival of the population. Therefore, these models exhibit Parrondo's paradox, an abstraction of the phenomenon of flashing Brownian ratchets [23-25], where a winning outcome can be achieved by alternating between two losing strategies [26-29].

Inspired by the previous analyses of the predator-prey model, we propose a population dynamic model to investigate the competition between two forms of prey with different settings under predation. The two forms of dormitive prey $y_{1}$ and $y_{2}$ are both losing strategies compared with the perennially active (PA) prey $p$ with superior traits. Indeed, the active form of the dormitive prey $y_{1}$ has a lower growth rate than the PA prey, spending energy on dormant offspring rather than foraging, and thus loses; the dormant form $y_{2}$ is also a losing strategy due to its low growth rate and inability to grow on its own. In the game-theoretic perspective, these two strategies cannot individually compete with the PA prey. However, alternating between these two losing strategies allows the dormitive prey to gain an advantage in the competition. This result recapitulates the phenomenon of Parrondo's paradox. An additional unexpected finding is that the dormitive prey, which generally would be assumed to be more effective under harsh environmental conditions, in this model, has a higher fitness in richer environments. This outcome is determined by the high density of predators and the large-amplitude fluctuations.

\section{Results}

The competition between different forms of prey and the effect of dormancy

We develop a population dynamic model to explore the competition between the two types of prey under predation (Eqs. 1, 2, and 3). The differences between the two types of prey are that (1) the PA prey $p$ has a higher growth rate $r_{p}$ and (2) the dormitive prey has two forms (active form $y_{1}$ and dormant form $y_{2}$ ) which are determined by dormancy switching function $\mu(z)$ and dormancy termination rate $\alpha$. In general, the prey with a higher growth rate will dominate and survive for a long time in the environment under predation. Hence, the density of the predator $z$ is one of the vital parameters for the competition of prey. Especially, the predator density determines the dormancy switching in our model such that there is more dormant form under a higher predator density but more active form under a lower density of predators. Here, we assume that the density of the dormant form of the dormitive prey does not contribute to the density-dependent growth, and the mortality rate is a constant for the predator. In gametheoretic terms, the active form $y_{1}$ and dormant form $y_{2}$ 
of the dormitive prey are both losing strategies compared with the PA prey $p$ because (1) the active form has a lower growth rate and spends extra energy to produce dormant offspring and (2) the dormant form also has a lower growth rate and cannot support population growth on its own. However, will the dormitive prey with a lower growth rate but capable of entering dormancy under harsh environmental conditions overtake the PA prey and win the competition in the environment? The detailed descriptions, the initial values, and the units of parameters and functions used in our model, which were obtained from published real-world data [11, 14, 30-32], are given in Table 1.

In the first competition scenario, we assume that the active form or the dormant form of the dormitive prey competes with the PA prey individually (Fig. 1A, B), under different initial population densities, dormancy termination rates $\alpha$, and dormancy switching functions $\mu(z)$. In this case, $y_{2}$ equals 0 (Fig. 1A), and $y_{1}$ equals 0 (Fig. 1B) individually. In this competition, the active form of the dormitive prey is disadvantaged compared with the PA prey and eventually goes extinct $(t=40)$ due to its low growth rate (Fig. 1A), whereas the dormant form of the dormitive prey becomes extinct already in the first wave because it cannot sustain population growth on its own (Fig. 1B). Thus, both forms are losing strategies for the dormitive prey, so that the PA prey outcompetes $y$ under all conditions. In this competition, the interaction between the PA prey and the predator follows the Rosenzweig-MacArthur criterion [33].

In the next competition scenario, we assume that the dormitive prey can switch between the active and dormant forms (Fig. 1C) due to the setting of $\mu(z)$. In this case, the PA prey is dominant at the beginning $(t \in[0,320])$ thanks to its high growth rate $r_{p}$. However, the PA prey cannot support the high density of predators. At high density, the predator will forage for a large number of the PA prey, causing its extinction, because it has no recourse to an evasive strategy (dormancy). Thus, it begins to decline after $t=200$ and becomes extinct at $t=380$. Then, the dormitive prey overtakes the PA prey and gradually evolves towards a steady state of coexistence with the predator. There are more active forms at lower density of the predator and more dormant forms under higher density of predators because of the setting of $\mu(z)$. The density of the active form is similar to the density of the PA prey, which is determined by the carrying capacity $K$. In order to examine the dynamics of different populations more closely, a cycle of the dynamic process $(t \in[400,450])$ is enlarged (Fig. 1D). The peaks of different populations do not occur at the same time. Specifically, the peak time of $y_{2}$ occurs between the peak times of $y_{1}$ and $z$. In addition, the growth rate of the dormant form of the dormitive prey around $t=420$ is higher than the rate during $t \in[410$, 418], which is determined by the growth term $r_{y}\left(1-\frac{p+y_{1}}{K}\right)($ $1-\mu(z)) y_{1}$. During $t \in[410,418]$, the growth of the dor-

Table 1 The descriptions, values, and units of parameters and functions used in the model (from recent real-world data [11, 14, 30-32])

\begin{tabular}{|c|c|c|c|}
\hline Parameter & Description & Value & Units \\
\hline $\bar{p}$ & Perennially active prey density & 2 & $\mathrm{mg} \mathrm{L}^{-1}$ \\
\hline$y_{1}$ & Dormitive prey (active form) density & 2 & $\mathrm{mg} \mathrm{L}^{-1}$ \\
\hline$y_{2}$ & Dormitive prey (dormant form) density & 0 & $\mathrm{mg} \mathrm{L}^{-1}$ \\
\hline$z$ & Predator density & 1 & $\mathrm{mg} \mathrm{L}^{-1}$ \\
\hline$r_{p}, r_{y}$ & Prey growth rate & $0.55,0.5$ & $\mathrm{mg} \mathrm{L}^{-1}$ \\
\hline K & Prey carrying capacity & 11 (Variable) & $\mathrm{mg} \mathrm{L}^{-1}$ \\
\hline$d_{z}$ & Predator death rate & 0.2 & day $^{-1}$ \\
\hline$a$ & Dormancy termination rate & 0.05 & $\operatorname{day}^{-1}$ \\
\hline$k_{p}, k_{y_{1}}$ & Predator growth efficiencies (from active form) & 0.5 & - \\
\hline$k_{y_{2}}$ & Predator growth efficiency (from dormant form) & 0.25 & - \\
\hline$c_{p}, c_{y_{1}}, c_{y_{2}}$ & Predator foraging efficiencies & $1,1,0.4$ & day $^{-1} \mathrm{mg}^{-1} \mathrm{~L}$ \\
\hline$h_{p}, h_{y_{1}}, h_{y_{2}}$ & Predator handling times & 0.5 & day \\
\hline$\eta$ & Dormancy switching threshold & 1 & $\mathrm{mg} \mathrm{L}^{-1}$ \\
\hline$\sigma$ & Dormancy switching width & 0.1 & $\mathrm{mg} \mathrm{L}^{-1}$ \\
\hline$x$ & Lower bound & 0.2 & $\mathrm{mg} \mathrm{L}^{-1}$ \\
\hline$\varphi$ & Range & 0.75 & $\mathrm{mg} \mathrm{L}^{-1}$ \\
\hline$f_{p}(p), f_{y_{1}}\left(y_{1}\right), f_{y_{2}}\left(y_{2}\right)$ & Predation rates & Function & day $^{-1}$ \\
\hline$\mu(z)$ & Dormancy switching function & Function & - \\
\hline
\end{tabular}



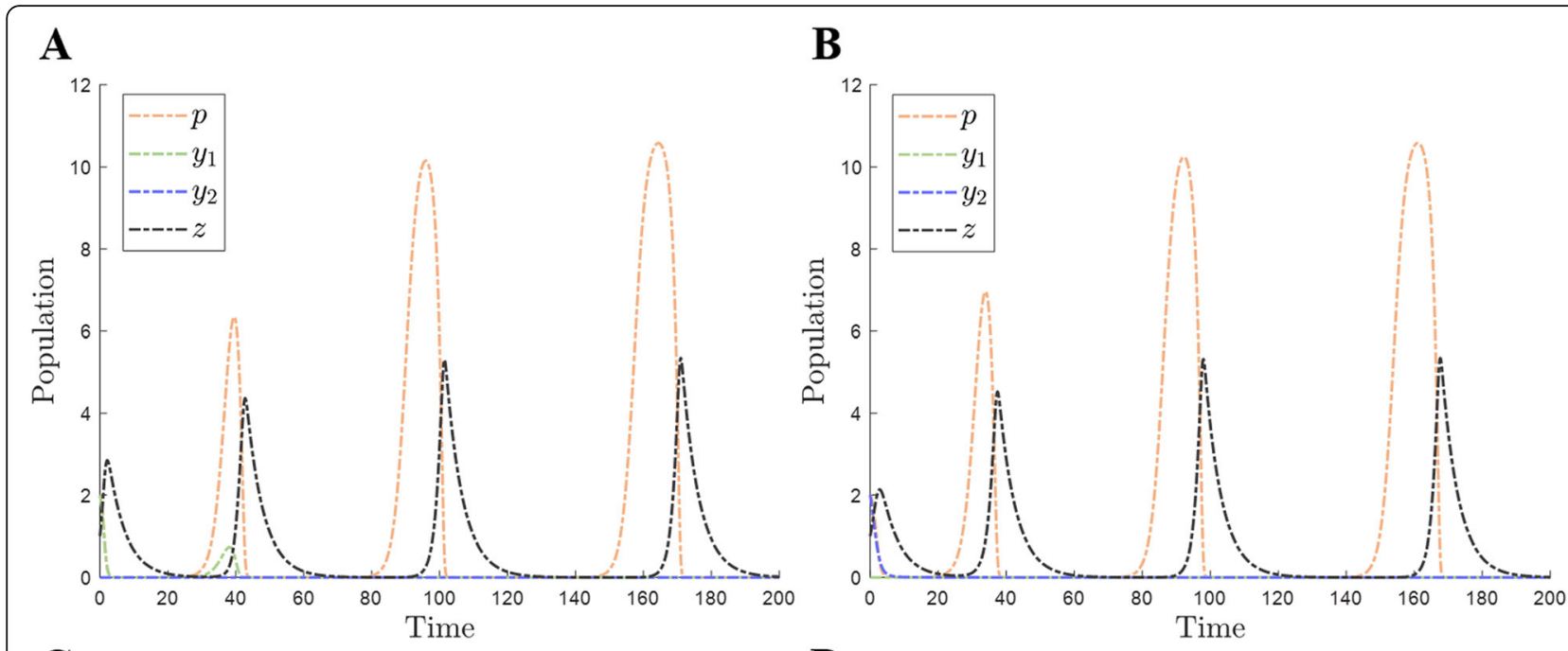

C

D
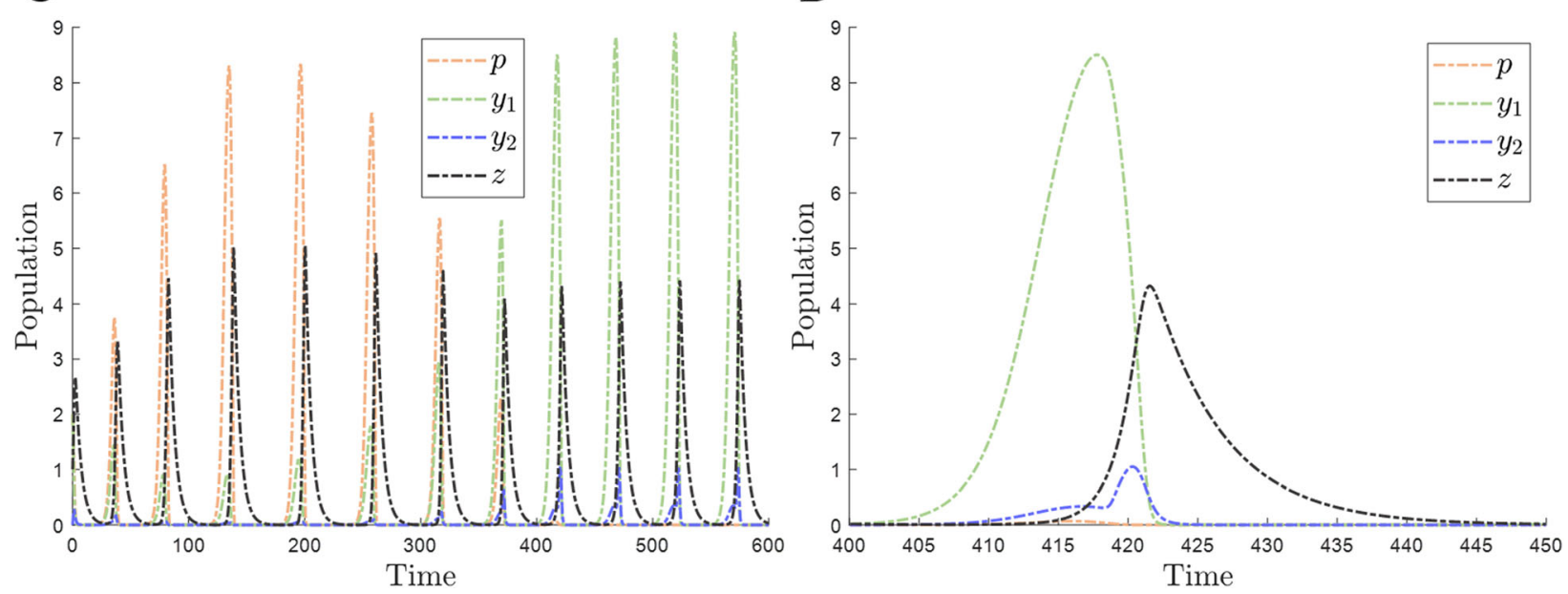

Fig. 1 Competition between two types of prey. The parameter values are from Table 1 unless stated otherwise. A Only the active form of the dormitive prey is present, in competition with the PA prey. The initial population density is $[2,2,0,1], a=1, X=1$, and $\varphi=0$. B Only the dormant form of the dormitive prey is present, in competition with the PA prey. The initial population density is $[2,0,2,1], a=0, X=0$, and $\varphi=0$. C Both the active and dormant forms of the dormitive prey are present, in competition with the PA prey. The initial population density is $[2,2,0,1]$. $\mathbf{D}$ A segment of the dynamics in $\mathbf{C}$ is enlarged $(t \in[400,450])$

mant form is mainly due to the increasing density of the active form of the dormitive prey, causing the increase of ( $\left.1-\frac{p+y_{1}}{K}\right) y_{1}$, under the logistic growth model. However, after $t=418$, the density of the active form begins to drop and the density of the predator begins to increase, and therefore, the growth rate of the dormant form is higher than before because of the rapid increase in $1-\mu(z)$ (Eq. $3)$. After $t=420$, the growth rate is slower than the foraging rate, so the dormant form of the dormitive prey declines and the predator reaches its peak.

\section{Impact of the carrying capacity on the outcome of the competition}

As the carrying capacity $K$ increases, there will be more prey, causing a higher density of predators. However, our previous results show that the PA prey cannot support the high density of the predator and thus becomes extinct. Hence, the impact of the carrying capacity on the competition between the two types of prey should be studied. Compared with $K=11$ (Fig. 1C), the dormitive prey will take advantage earlier at $K=13$ (Fig. 2A). The PA prey cannot reach its peak (near carrying capacity) under these conditions and will begin to decline at a point where it still has an apparent potential to rise. This is the case because the current density of prey already results in high predator density such that the prey cannot reach the environmental capacity due to the high rate of predation. However, the predator grows faster than before thanks to the availability of adequate resources, so the PA prey will become extinct quickly because of the lack of an evasive strategy. The first time when the peak density of the active form of the 


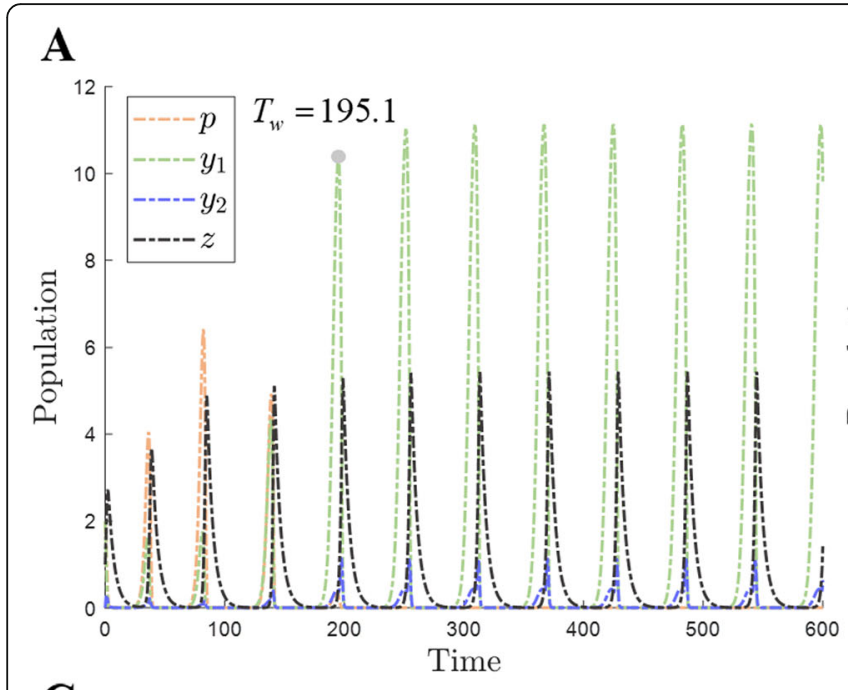

\section{B}
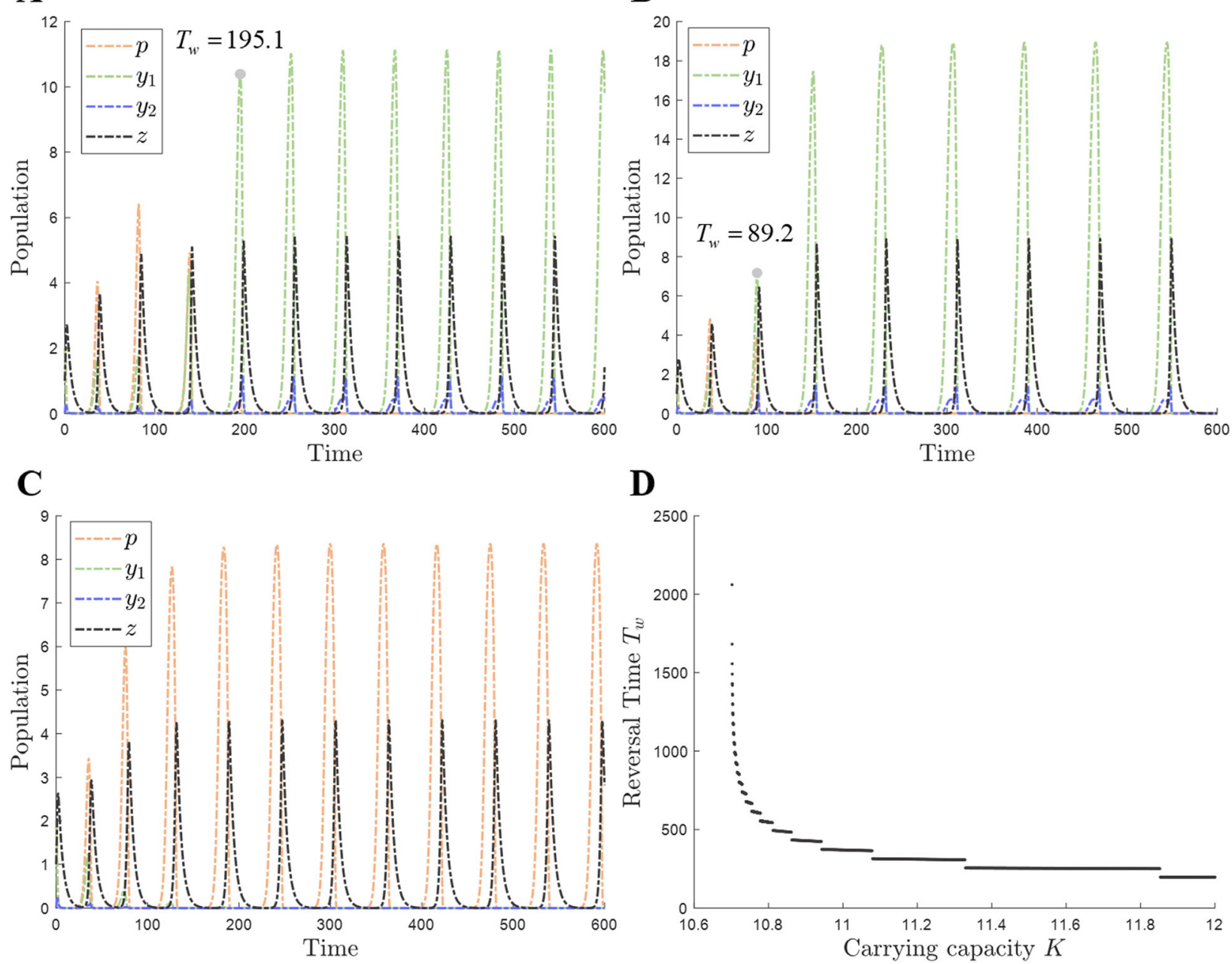

D

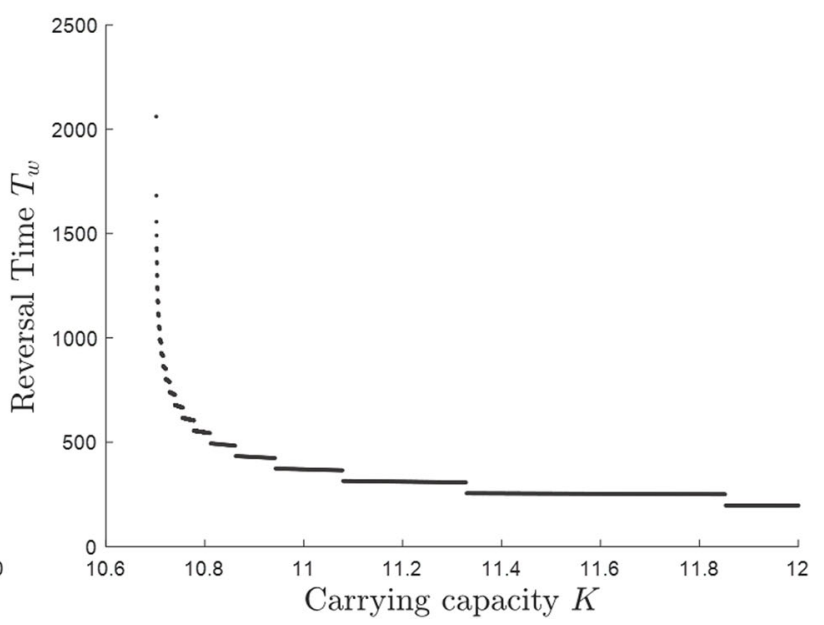

Fig. 2 Competition between two types of prey under different carrying capacities. The parameter values are from Table 1 unless stated otherwise. The initial population density is $[2,2,0,1]$. A Carrying capacity $K$ equals 13 . B Carrying capacity $K$ equals 20 , causing higher density of each population and shorter reversal time $T_{w}$ compared to the case that $K$ equals 13 . C Carrying capacity $K$ equals 9 , the PA prey wins the competition. D The relationship between the reversal time $T_{w}$ and carrying capacity $K$.

dormitive prey is higher than the peak density of the PA prey is called the reversal time $T_{w}$. The reversal time $T_{w}$ is 195.1 in this case (Fig. 2A), that is, reversal occurs earlier than it does at $K=11$. When $K$ continues to rise to 20 (Fig. 2B), the reversal time further shortens, to $T_{w}=89.2$. In addition, the peak density of the active form of the dormitive prey is also higher than for the previous conditions and comes close to the carrying capacity. In contrast, when $K$ decreases to 9 (Fig. 2C), the PA prey is at an advantage because the density of the predator is lower than it is at larger $K$. This allows the PA prey to grow in the environment and avoid extinction. The peak density of the PA prey is also close to $K=9$, the same as discussed before. At a high predator density, the PA prey will be extensively foraged and will eventually go extinct. In contrast, the dormitive prey can enter dormancy to survive at a high density of the predator, which is a competitive survival strategy. Hence, the dormant form is advantageous to the prey under population fluctuation in the apparent competition [34]. The reversal time $T_{w}$ changes rapidly when the carrying capacity $K$ is low but stays nearly constant at high $K$ (Fig. 2D).

\section{Analysis of the parameter space}

We further explored the parameter space of the model to determine how the dormitive prey gains advantage under different conditions. The outcomes were evaluated by the highest density of each competitor during $t \in[2800,3000]$ (stable competition). Due to the impracticality of all-vs-all parameter comparison, the parameters were analyzed in pairs as follows. 


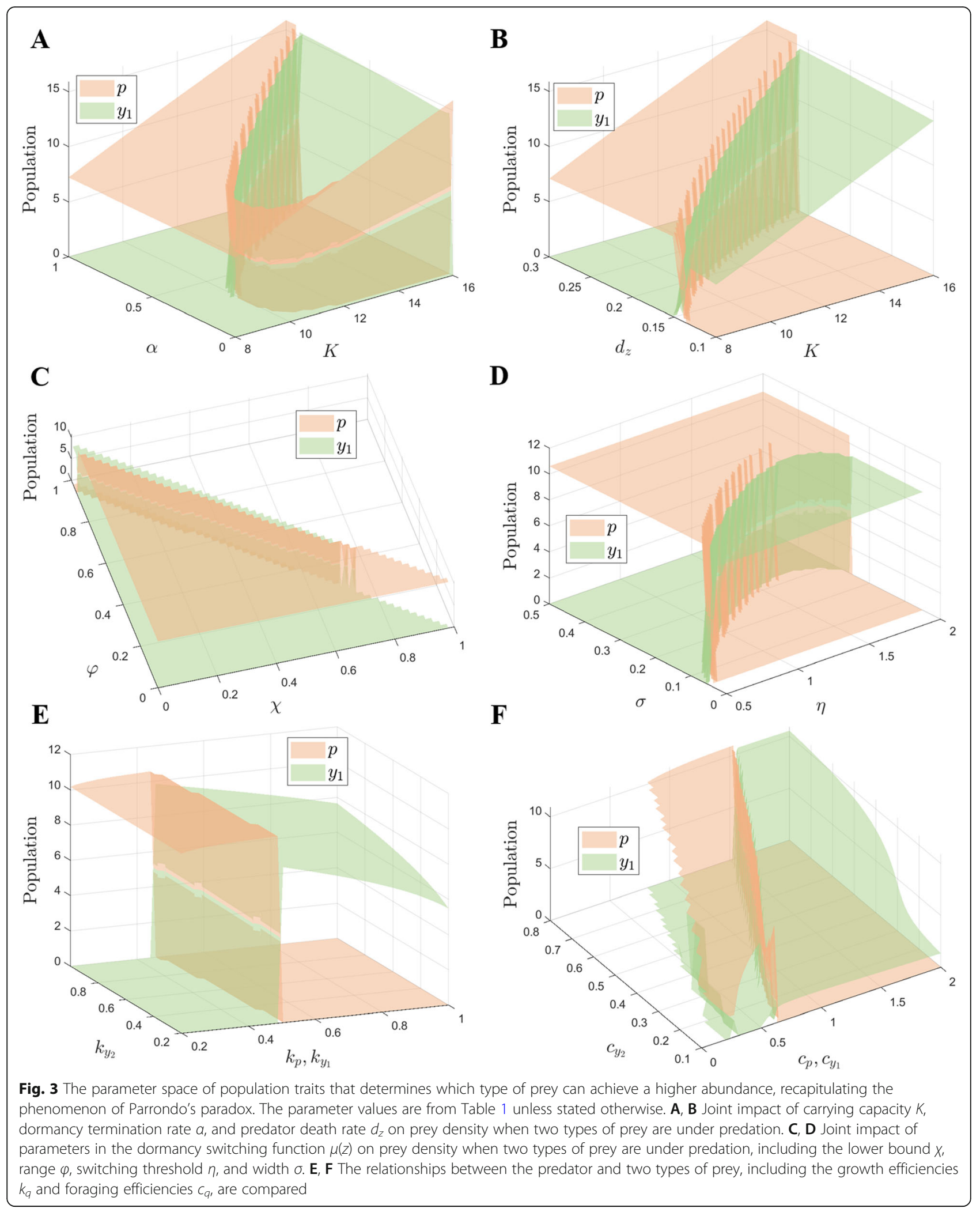


The carrying capacity $K$ was first compared with the dormancy termination rate $\alpha$ (Fig. 3A). When $K$ is low, the PA prey reaches a higher abundance than the active form of the dormitive prey, no matter how $\alpha$ changes. This is because there are not enough predators to consume resources (prey) under low $K$, thus avoiding the extinction of the PA prey. However, as $K$ increases, a large number of predators will forage for the PA prey, leading to its extinction. In this case, the active form of the dormitive prey gains advantage under medium values of $\alpha$, because both the high and low $\alpha$ only allow a single form of dormitive prey to exist, which are losing strategies compared with the PA prey (Fig. 1A, B). The carrying capacity $K$ is then compared with the predator death rate $d_{z}$ (Fig. 3B). With the increase of $K$, the density of both types of prey will increase, the same as the result in Fig. 3A. At low $K$, only small values of $d_{z}$ allow the active form of the dormitive prey to win because only the dormitive prey can survive under the high density of predators in this case. There will be more prey in the environment with higher $K$, leading to many predators; thus, the PA prey can only win under higher values of $d_{z}$ (fewer predators) as shown above. Hence, there is only a narrow range of $d_{z}$ for the PA prey to dominate in the environment under high $K$, but a wide range of $d_{z}$ under low $K$.

Four parameters in the dormancy switching function $\mu(z)$ were then compared because of the importance of switching for the dormitive prey. The lower bound $X$ and range $\phi$ determine how many preys remain active and how many go dormant (Fig. 3C). The values of $\chi$ and $\phi$ obey the condition $0 \leq X \leq X+\phi \leq 1$, which means that the value of the dormancy switching function should be between 0 and 1 . We find that the active form of the dormitive prey can only gain advantage when $X$ is relatively low $(x \leq 0.6)$ and $X+\phi$ is close to 1 . $X$ cannot be too large because the prey needs a certain amount of active form to provide the growth capacity in all cases; otherwise, the dormitive prey will go extinct. The reason why $X+\phi \approx 1$ is to ensure full reproduction under safe conditions, so that the dormitive prey can survive under environmental fluctuations. In the comparison of switching threshold $\eta$ and width $\sigma$ in $\mu(z)$ (Fig. 3D), the dormitive prey can reach a higher abundance than the PA prey with low $\sigma$ because of the sharp switching. The sharp switching between the active and dormant form results in a strong fitness to the environment, allowing the disadvantaged prey to win in the competition.

The relationships between the predator and two types of prey are further studied. The comparison between the predator growth efficiencies from the active form $k_{p}, k_{y_{1}}$ and the efficiency from the dormant form $k_{y_{2}}$ (Fig. 3E) shows that $k_{y_{2}}$ does not affect the result of the competition. Higher $k_{p}$ and $k_{y_{1}}$ will cause more predators in the environment, resulting in the winning outcome of the active form of the dormitive prey (similar results in Fig. 3B). We assume the foraging efficiencies $c_{y_{2}} \leq c_{p}$ and $c_{y_{2}} \leq c_{y_{1}}$ in Fig. 3F because the dormant form is less likely to be found and thus avoids predation. There will be more predators under higher $c_{p}$ and $c_{y_{1}}$, and thus, it can be concluded that the dormitive prey will win in this case (same reason as in Fig. 3B).

\section{Discussion}

Analysis of the present model shows that the dormitive prey can outcompete the PA prey with superior traits by switching between the active and dormant forms. The counterintuitive results obtained here are that (1) the dormitive prey with lower growth rate and extra energy loss can overtake the PA prey and win the competition and (2) prey dormancy is advantageous in rich environments (high carrying capacity), whereas intuitively, it could be expected to gain advantage in poor environments. These surprising outcomes of the competition can be explained through Parrondo's paradox: alternation of two losing strategies can result in a winning strategy. In this model, both the active form and the dormant form of the dormitive prey are individually disadvantaged in the competition against the PA prey because (1) the active form of the dormitive prey has a lower growth rate and spends extra energy to produce dormant offspring, and (2) the dormant form also has a lower growth rate and cannot support population growth on its own (Fig. 1A, B). However, a winning outcome for the dormitive prey can be achieved by alternating between the active form and the dormant form (Fig. 1C). This result can be explained by Parrondo's paradox [35-37]. Parrondo's paradox also might be applicable to other biological competitions. For example, bacteriophages can switch between active reproduction (hostdestroying lytic phase) and dormancy (lysogenic phase) depending on the state of the external environment [38], and the dormancy of phages (lysogeny) can be considered an adaptation to host population oscillations.

Under a high carrying capacity, a high density of prey is reached by logistic growth [39], thereby supporting a high density of predators. However, the high density of predators will lead to the extinction of the PA prey because the latter has no recourse to dormancy as an evasive strategy (Fig. 2A, B). Hence, the dormitive prey has higher fitness under higher carrying capacity (higher density of predators). Furthermore, the higher the carrying capacity, the sooner (smaller value of the reversal time $T_{w}$ ) the dormitive prey will gain advantage and overtake (Fig. 2D) because predators will grow faster and forage more PA prey. In contrast, the PA prey will gain advantage under the low peak density of predators (Fig. 
2C) because it can support the predator population at this low density. Therefore, the density of predators (determined by the carrying capacity in our model) is the key factor that determines the outcome of the competition between the two types of prey [34, 40]. This allows us to interpret the second counterintuitive result: prey dormancy is advantageous in richer environments. The resource-rich environment not only increases the density of the prey, but also causes the large-amplitude population (predator-prey) cycles and high density of predators due to the prey oversupply, followed by over-predation $[15,17]$. The dormant form of prey can survive under the high density of predators in the population cycles to come back subsequently, whereas the PA prey goes extinct in the cycles. Furthermore, no matter which prey has the advantage in the competition, the peak density of prey is higher in a richer environment, approaching the carrying capacity and causing large amplitude cycles, which is beneficial to the dormitive prey.

The large-amplitude population cycles have been explored in diverse real-world cases, such as the relationship between Daphnia and plankton [15, 41], Arctic lemmings and weasels [42], parasites and mountain hares [43], and intraspecific competition of Antarctic krill [44]. These results show the effect of over-predation and potential applications of Parrondo's paradox $[45,46]$. The large-amplitude fluctuations are usually accompanied by the extinction of populations $[16,17]$, but dormancy allows the prey to survive the fluctuation, with a subsequent comeback. Thus, our key finding is that the dormitive prey can gain advantage and overtake in the competition under large-amplitude fluctuations. Indeed, prey dormancy has been observed in a variety of predator-prey systems including bacteria (Myxococcus as predator and Bacillus as prey) [47-49]; arthropods-spider mites [50, 51]; and small rodents-dormice [52, 53].

An obvious limitation of this work is that, because of the wide variation of the parameters of predator and prey populations, the dynamics derived from the real-world data [11, 14, 30-32] might substantially differ from those predicted by the model. Nevertheless, we explored in detail the impact of multiple parameter combinations on the competition outcome. In particular, this analysis shows that the parameter values that cause a higher density of predators, such as lower death rate, higher predator growth efficiencies, and higher foraging efficiencies, will lead to the extinction of the PA prey. In addition, the range of dormancy termination rates ensuring the winning outcome for the dormitive prey is determined by the carrying capacity, which can also affect the density of predators. The parameters in the threshold-induced dormancy function also need to be in a suitable range for the dormitive prey to win the competition.

\section{Conclusion}

The analysis of the present model indicates that the counterintuitive winning outcome for the dormitive prey is underlain by the game-theoretic Parrondo's paradox. In the large-amplitude fluctuation, dormancy can help the dormitive prey to outcompete the PA prey with superior traits and even cause the extinction of the PA prey. Parrondo's paradox is likely to be widely applicable to other biological competitions with large-amplitude fluctuations.

\section{Methods}

\section{Population model}

In the dynamic population model, we introduce an additional prey population $(y)$ to the existing predator-prey model, adapted from the Lotka-Volterra-derived model of Rosenzweig and MacArthur [33, 54, 55]. In detail, two prey populations $(p, y)$ are both preyed on by the predator population $(z) \cdot p$ is the density of PA prey, and $y=$ $y_{1}+y_{2}$ is the density of dormitive prey, with $y_{1}$ and $y_{2}$ corresponding to the active and dormant subpopulations, respectively. Here, we assume that (1) the dormant form of the dormitive prey is sterile, (2) the dormant form does not contribute to density-dependent growth regulation, and (3) density-dependence applies to growth but not to mortality. The differential equations of this proposed model are:

$$
\begin{aligned}
& \dot{p}=r_{p}\left(1-\frac{p+y_{1}}{K}\right) p-f_{p}(p) z, \\
& y_{1}=r_{y}\left(1-\frac{p+y_{1}}{K}\right) \mu(z) y_{1}+\alpha y_{2}-f_{y_{1}}\left(y_{1}\right) z, \\
& \dot{y}_{2}=r_{y}\left(1-\frac{p+y_{1}}{K}\right)(1-\mu(z)) y_{1}-\alpha y_{2}-f_{y_{2}}\left(y_{2}\right) z, \\
& \dot{z}=k_{p} f_{p}(p) z+k_{y_{1}} f_{y_{1}}\left(y_{1}\right) z+k_{y_{2}} f_{y_{2}}\left(y_{2}\right) z-d_{z} z .
\end{aligned}
$$

Two types of prey follow the logistic growth model [56] with the environment carrying capacity $K$ and different maximum growth rates $\left(r_{p}, r_{y}\right)$, as well as the predation from the predator at rates $f_{p}(p), f_{y_{1}}\left(y_{1}\right)$, and $f_{y_{2}}\left(y_{2}\right)$ (Eq. 2). The active form is also supplemented by the dormant form at the termination rate $\alpha$, and only a fraction of energy, $\mu(z)$, is used for the active descendant of the dormitive prey. Therefore, the remaining fraction, $1-\mu(z)$, is used for the dormant progeny. The predator grows in proportion to the growth efficiencies $\left(k_{p}, k_{y_{1}}\right.$, $\left.k_{y_{2}}\right)$ and predation rates $\left(f_{p}(p), f_{y}\left(y_{1}\right), f_{y_{2}}\left(y_{2}\right)\right)$, while dying at rate $d_{z}$. Recent published real-world data [11, 14, 3032] shown in Table 1 are used to validate our proposed dynamic model. These data have been applied to 
describe the traits of prey and predator in the competition from the zooplankton modeling literature. Numerical simulations were implemented by the ode 23 function in MATLAB that is an implementation of an explicit Runge-Kutta $(2,3)$ pair of Bogacki and Shampine [57]. The accuracy of the simulation is ensured by the strict tolerance level in the repeated experiment, which makes the final coefficient not change significantly and less than $1 \%$. In the simulation, the relative error tolerance and absolute error tolerance are both $10^{-8}$.

\section{Parameter functions}

The predation function $f_{q}(q)$ originated from the Holling type II function response, a monotone increasing function based on the property of prey:

$$
f_{q}(q)=\frac{c_{q} q}{1+c_{q} h_{q} q}, q \in\left\{p, y_{1}, y_{2}\right\}
$$

where $c_{q}$ and $h_{q}$ are the foraging efficiency and handling time, respectively. There are different values of parameters for different types of prey. $f_{q}(q)$ equals to $c_{q} q$ and $1 /$ $h_{q}$ when $q \rightarrow 0$ and $q \rightarrow \infty$, respectively.

The dormancy switching function $\mu(z)$ is obtained by the improved sigmoid switching function (monotonically decreasing) based on the predator density $z$ :

$$
\mu(z)=x+\phi\left[1+\exp \left(\frac{z-\eta}{\sigma}\right)\right]^{-1},
$$

where $X$ and $\phi$ are the lower bound and range of the function, and $\eta$ and $\sigma$ denote the switching threshold and shape of the switching function, respectively.

\section{Abbreviation}

PA: Perennially active

\section{Acknowledgements}

Not applicable.

\section{Authors' contributions}

K. H. C. and E. V. K. designed the research study. T. W. and K. H. C. performed the research. T. W. wrote the code. T. W., K. H. C., and E. V. K. analyzed the data. T. W., K. H. C., and E. V. K. wrote the paper. K. H. C. supervised the study. All authors read and approved the final version of the paper.

\section{Funding}

T. W. and K. H. C. are supported by the Singapore University of Technology and Design (Grant No. SRG SCI 2019 142). E. V. K. is supported by the Intramural Research Program of the National Institutes of Health of the USA (National Library of Medicine).

\section{Availability of data and materials}

MATLAB code used for this paper is available at https://osf.io/74agb/?view_ only=006d473342d0455f9b3addc3164fd644 [58].

\section{Declarations}

Ethics approval and consent to participate

Not applicable.

\section{Consent for publication}

Not applicable.

\section{Competing interests}

The authors declare that they have no competing interests.

\section{Author details}

${ }^{1}$ Science, Mathematics and Technology Cluster, Singapore University of Technology and Design (SUTD), 8 Somapah Road, S487372, Singapore, Singapore. ${ }^{2}$ National Center for Biotechnology Information, National Library of Medicine, National Institutes of Health, Bethesda, MD 20894, USA.

Received: 26 February 2021 Accepted: 14 July 2021

Published online: 23 August 2021

\section{References}

1. Geiser $F$, Turbill C. Hibernation and daily torpor minimize mammalian extinctions. Naturwissenschaften. 2009;96(10):1235-40. https://doi.org/10.1 007/s00114-009-0583-0.

2. Kobbe S, Dausmann $\mathrm{KH}$. Hibernation in Malagasy mouse lemurs as a strategy to counter environmental challenge. Naturwissenschaften. 2009; 96(10):1221-7. https://doi.org/10.1007/s00114-009-0580-3.

3. Gyllström M, Hansson L-A. Dormancy in freshwater zooplankton: induction, termination and the importance of benthic-pelagic coupling. Aquat Sci. 2004;66(3):274-95.

4. Leck MA. Ecology of soil seed banks: Elsevier; 2012.

5. Lennon JT, Jones SE. Microbial seed banks: the ecological and evolutionary implications of dormancy. Nat Rev Microbiol. 2011;9(2):119-30. https://doi. org/10.1038/nrmicro2504.

6. Li L, Mendis N, Trigui H, Oliver JD, Faucher SP. The importance of the viable but non-culturable state in human bacterial pathogens. Front Microbiol. 2014;5:258.

7. Ramamurthy T, Ghosh A, Pazhani GP, Shinoda S. Current perspectives on viable but non-culturable (VBNC) pathogenic bacteria. Front Public Health. 2014;2:103.

8. Setlow P, Christie G. Bacterial spore mRNA-what's up with that? Front Microbiol. 2020;11:2597.

9. Clauss $M$, Venable $D$. Seed germination in desert annuals: an empirical test of adaptive bet hedging. Am Nat. 2000;155(2):168-86. https://doi.org/10.1 086/303314.

10. Hairston NG Jr, Kearns CM. Temporal dispersal: ecological and evolutionary aspects of zooplankton egg banks and the role of sediment mixing. Integr Comp Biol. 2002;42(3):481-91. https://doi.org/10.1093/icb/42.3.481.

11. Freire JG, Gallas MR, Gallas JA. Impact of predator dormancy on preypredator dynamics. Chaos. 2018;28(5):053118. https://doi.org/10.1063/1.501 6434.

12. Waterkeyn A, Vanoverbeke J, Van Pottelbergh N, Brendonck L. While they were sleeping: dormant egg predation by Triops. J Plankton Res. 2011; 33(10):1617-21. https://doi.org/10.1093/plankt/fbr048.

13. Kuwamura M, Nakazawa T. Dormancy of predators dependent on the rate of variation in prey density. SIAM J Appl Math. 2011;71(1):169-79. https://doi. org/10.1137/100781985.

14. Tan ZX, Koh JM, Koonin EV, Cheong KH. Predator dormancy is a stable adaptive strategy due to Parrondo's paradox. Adv Sci. 2020;7(3):1901559. https://doi.org/10.1002/advs.201901559.

15. McCauley E, Nisbet RM, Murdoch WW, de Roos AM, Gurney WS. Largeamplitude cycles of Daphnia and its algal prey in enriched environments. Nature. 1999:402(6762):653-6. https://doi.org/10.1038/45223.

16. Kuwamura M, Nakazawa T, Ogawa T. A minimum model of prey-predator system with dormancy of predators and the paradox of enrichment. J Math Biol. 2009;58(3):459-79. https://doi.org/10.1007/s00285-008-0203-1.

17. Rosenzweig ML. Paradox of enrichment: destabilization of exploitation ecosystems in ecological time. Science. 1971;171(3969):385-7. https://doi. org/10.1126/science.171.3969.385.

18. Eilersen A, Sneppen K. The uneasy coexistence of predators and pathogens. Eur Phys J E. 2020;43(7):1-7.

19. Eilersen A, Sneppen K. Applying allometric scaling to predator-prey systems. Phys Rev E. 2019;99(2):022405. https://doi.org/10.1103/PhysRevE.99.022405.

20. Tan ZX, Cheong KH. Nomadic-colonial life strategies enable paradoxical survival and growth despite habitat destruction. eLife. 2017;6:e21673. https://doi.org/10.7554/eLife.21673.

21. Cheong KH, Tan ZX, Ling YH. A time-based switching scheme for nomadiccolonial alternation under noisy conditions. Commun Nonlinear Sci Numer Simul. 2018;60:107-14. https://doi.org/10.1016/j.cnsns.2017.12.012. 
22. Koh JM, Xie N-g, Cheong KH. Nomadic-colonial switching with stochastic noise: subsidence-recovery cycles and long-term growth. Nonlinear Dynamics. 2018;94(2):1467-77. https://doi.org/10.1007/s11071-018-4436-2.

23. Cao FJ, Dinis L, Parrondo JM. Feedback control in a collective flashing ratchet. Phys Rev Lett. 2004;93(4):040603. https://doi.org/10.1103/ PhysRevLett.93.040603.

24. Harmer GP, Abbott D. Losing strategies can win by Parrondo's paradox. Nature. 1999;402(6764):864-864. https://doi.org/10.1038/47220.

25. Rousselet J, Salome L, Ajdari A, Prostt J. Directional motion of Brownian particles induced by a periodic asymmetric potential. Nature. 1994; 370(6489):446-7. https://doi.org/10.1038/370446a0.

26. Cheong $\mathrm{KH}$, Koh JM, Jones MC. Paradoxical survival: examining the Parrondo effect across biology. BioEssays. 2019;41(6):1900027. https://doi. org/10.1002/bies.201900027.

27. Lai JW, Chang J, Ang L, Cheong KH. Multi-level information fusion to alleviate network congestion. Inf Fusion. 2020;63:248-55. https://doi.org/10.1 016/j.inffus.2020.06.006.

28. Williams PD, Hastings A. Paradoxical persistence through mixed-system dynamics: towards a unified perspective of reversal behaviours in evolutionary ecology. Proc R Soc B Biol Sci. 2011;278(1710):1281-90. https:// doi.org/10.1098/rspb.2010.2074

29. Koh JM, Cheong KH. Generalized solutions of Parrondo's games. Adv Sci. 2020;7(24):2001126. https://doi.org/10.1002/advs.202001126.

30. Kuwamura M, Chiba H. Mixed-mode oscillations and chaos in a preypredator system with dormancy of predators. Chaos. 2009;19(4):043121. https://doi.org/10.1063/1.3270262.

31. Rose KA, Swartzman GL, Kindig AC, Taub FB. Stepwise iterative calibration of a multi-species phytoplankton-zooplankton simulation model using laboratory data. Ecol Model. 1988;42(1):1-32. https://doi.org/10.1016/0304-3 800(88)90089-0.

32. Scheffer M, De Boer RJ. Implications of spatial heterogeneity for the paradox of enrichment. Ecology. 1995;76(7):2270-7. https://doi.org/10.2307/1941701.

33. Rosenzweig ML, MacArthur RH. Graphical representation and stability conditions of predator-prey interactions. Am Nat. 1963;97(895):209-23. https://doi.org/10.1086/282272.

34. Holomuzki JR, Feminella JW, Power ME. Biotic interactions in freshwater benthic habitats. J North Am Benthological Soc. 2010;29(1):220-44. https:// doi.org/10.1899/08-044.1.

35. Cheong KH, Koh JM, Jones MC. Multicellular survival as a consequence of Parrondo's paradox. Proc Natl Acad Sci. 2018;115(23):E5258-9. https://doi. org/10.1073/pnas.1806485115.

36. Cheong KH, Tan ZX, Xie N-g, Jones MC. A paradoxical evolutionary mechanism in stochastically switching environments. Sci Rep. 2016;6(1):1-12.

37. Cheong KH, Wen T, Lai JW. Relieving cost of epidemic by Parrondo's paradox: a COVID-19 case study. Adv Sci. 2020;7(24):2002324. https://doi. org/10.1002/advs.202002324.

38. Ptashne M. A genetic switch: phage lambda revisited: CSHL press; 2004

39. Pearl R, Reed LJ. On the rate of growth of the population of the United States since 1790 and its mathematical representation. Proc Natl Acad Sci U S A. 1920;6(6):275-88. https://doi.org/10.1073/pnas.6.6.275.

40. Kareva I, Karev G. Natural selection between two games with applications to game theoretical models of cancer. Bull Math Biol. 2019;81(7):2117-32 https://doi.org/10.1007/s11538-019-00592-2.

41. Marshall CT, Peters RH. General patterns in the seasonal development of chlorophyll a for temperate lakes. Limnol Oceanogr. 1989;34(5):856-67. https://doi.org/10.4319/lo.1989.34.5.0856.

42. MacLean S Jr, Fitzgerald B, Pitelka F. Population cycles in arctic lemmings: winter reproduction and predation by weasels. Arct Alp Res. 1974;6(1):1-12. https://doi.org/10.2307/1550365.

43. Newey S, Thirgood S. Parasite-mediated reduction in fecundity of mountain hares. Proc R Soc Lond B Biol Sci. 2004;271(suppl_6):S413-5.

44. Ryabov AB, de Roos AM, Meyer B, Kawaguchi S, Blasius B. Competitioninduced starvation drives large-scale population cycles in Antarctic krill. Nat Ecol Evol. 2017;1(7):1-8.

45. Hastings A. Transient dynamics and persistence of ecological systems. Ecol Lett. 2001;4(3):215-20. https://doi.org/10.1046/j.1461-0248.2001.00220.x.

46. Hastings A. Transients: the key to long-term ecological understanding? Trends Ecol Evol. 2004;19(1):39-45. https://doi.org/10.1016/j.tree.2003.09.007.

47. Müller S, Strack SN, Ryan SE, Kearns DB, Kirby JR. Predation by Myxococcus xanthus induces Bacillus subtilis to form spore-filled megastructures. Appl Environ Microbiol. 2015;81(1):203-10. https://doi.org/10.1128/AEM.02448-14.
48. Müller S, Strack SN, Ryan SE, Shawgo M, Walling A, Harris S, et al. Identification of functions affecting predator-prey interactions between Myxococcus xanthus and Bacillus subtilis. J Bacteriol. 2016;198(24):3335-44. https://doi.org/10.1128/JB.00575-16.

49. Müller S, Strack SN, Hoefler BC, Straight PD, Kearns DB, Kirby JR. Bacillaene and sporulation protect Bacillus subtilis from predation by Myxococcus xanthus. Appl Environ Microbiol. 2014;80(18):5603-10. https://doi.org/10.112 8/AEM.01621-14.

50. Kroon A, Veenendaal RL, Bruin J, Egas M, Sabelis MW. "Sleeping with the enemy" - predator-induced diapause in a mite. Naturwissenschaften. 2008; 95(12):1195-8. https://doi.org/10.1007/s00114-008-0442-4.

51. Lees AD. The physiology of diapause in arthropods, vol. 4: CUP Archive; 1955.

52. Bieber C, Ruf T. Summer dormancy in edible dormice (Glis glis) without energetic constraints. Naturwissenschaften. 2009;96(1):165-71. https://doi. org/10.1007/s00114-008-0471-z

53. Lebl K, Bieber C, Adamík P, Fietz J, Morris P, Pilastro A, et al. Survival rates in a small hibernator, the edible dormouse: a comparison across Europe. Ecography. 2011;34(4):683-92. https://doi.org/10.1111/j.1600-0587.2010. 06691.x.

54. Roughgarden J. Theory of population genetics and evolutionary ecology: an introduction; 1979.

55. Eilersen A, Jensen MH, Sneppen K. Chaos in disease outbreaks among prey. Sci Rep. 2020;10(1):1-7.

56. Kareva I, Karev G. From experiment to theory: what can we learn from growth curves? Bull Math Biol. 2018;80(1):151-74. https://doi.org/10.1007/s11 538-017-0347-5.

57. Bogacki P, Shampine LF. A 3 (2) pair of Runge-Kutta formulas. Appl Math Lett. 1989;2(4):321-5. https://doi.org/10.1016/0893-9659(89)90079-7.

58. Wen $\mathrm{T}$, Koonin EV, Cheong KH. An alternating active-dormitive strategy enables disadvantaged prey to outcompete the perennially active prey through Parrondo's paradox. 2021. OSF. https://osf.io/74agb/?view_only= 006d473342d0455f9b3addc3164fd644.

\section{Publisher's Note}

Springer Nature remains neutral with regard to jurisdictional claims in published maps and institutional affiliations.
Ready to submit your research? Choose BMC and benefit from:

- fast, convenient online submission

- thorough peer review by experienced researchers in your field

- rapid publication on acceptance

- support for research data, including large and complex data types

- gold Open Access which fosters wider collaboration and increased citations

- maximum visibility for your research: over $100 \mathrm{M}$ website views per year

At BMC, research is always in progress.

Learn more biomedcentral.com/submissions 